Relations industrielles

Industrial Relations

\title{
Out of the Sweatshop: The Struggle for Industrial Democracy, Leon Stein (ed.), New York, The New York Times Book, Co., 1977, 367 pp.
}

\section{Gilles Dussault}

Volume 33, numéro 2, 1978

URI : https://id.erudit.org/iderudit/028883ar

DOI : https://doi.org/10.7202/028883ar

Aller au sommaire du numéro

Éditeur(s)

Département des relations industrielles de l'Université Laval

ISSN

0034-379X (imprimé)

1703-8138 (numérique)

Découvrir la revue

Citer ce compte rendu

Dussault, G. (1978). Compte rendu de [Out of the Sweatshop: The Struggle for Industrial Democracy, Leon Stein (ed.), New York, The New York Times Book, Co., 1977, 367 pp.] Relations industrielles / Industrial Relations, 33(2), 379-380. https://doi.org/10.7202/028883ar

Tous droits réservés @ C Département des relations industrielles de l'Universite Laval, 1978
Ce document est protégé par la loi sur le droit d'auteur. L’utilisation des services d'Érudit (y compris la reproduction) est assujettie à sa politique d'utilisation que vous pouvez consulter en ligne.

https://apropos.erudit.org/fr/usagers/politique-dutilisation/ 
sure des conflits, etc. Ce qui nous amène à se demander si l'on ne va pas tout simplement se retrouver dorénavant avec deux séries portant sur la mesure du même phénomène mais qu'on ne pourra pas comparer.

Enfin si telle n'est pas leur préoccupation première, les auteurs ne manquent jamais l'occasion de faire les mises-engarde qui s'imposent au niveau de l'utilisation et l'interprétation de ces données dans l'analyse de problème divers. Ce point contribue davantage à faire de ce document un outil méthodologique fort précieux.

Jacques MERCIER

\section{Université Laval}

Out of the Sweatshop: The Struggle for Industrial Democracy, Leon Stein (ed.), New York, The New York Times Book, Co., 1977, 367 pp.

Cet ouvrage relate, à travers près de 170 textes, la difficile implantation du syndicalisme dans l'industrie du vêtement aux États-Unis. Le «combat pour la démocratie industrielle» que Leon Stein veut décrire est celui de l'International Ladies' Garment Workers' Union (ILGWU). Stein caractérise les principales étapes de l'évolution de l'ILGWU à l'aide de textes extraits d'articles de journaux, de rapports de commissions d'enquête, d'ouvrages historiques, de discours politiques, de romans, etc. Les textes sont regroupés en chapitres qui traitent d'événements ou de séquences d'événements spécifiques ou encore de thèmes généraux comme ceux de la difficulté de normaliser les salaires et les exigences de la production dans l'industrie du vêtement (chap. 12, "Pay and Production») et des problèmes de la syndicalisation des employés des entreprises très mobiles de ce genre d'industrie (chap. 13, «Old Battles, New Frontiers»). De nombreuses photographies et illustrations accentuent le réalisme de la description qui est faite des conditions de travail pénibles du secteur du vêtement.

Le «sweating system», dont la pratique est loin d'être disparue, s'est développé, aux États-Unis, grâce à une maind'œuvre docile et particulièrement vulnérable: celle des immigrants qui avaient fui des régimes autoritaires, comme celui de la Russie tsariste et qui étaient venus chercher en Amérique une liberté et une prospérité qui s'avèrent rapidement illusoires (chap. 2, "Sweatshop: Immigrant Style»). Un témoin de l'époque, Florence Kelley - qui fut le premier inspecteur d'usine de l'Etat d'Illinois - rapporte, en 1899 , qu'il n'y a à peu près pas d'américains d'origine dans le secteur du vêtement: "we never found any in sweatshops. The Native Americans who are working at the garment trades are perhaps the most dangerous of all from the point of view of the purchaser» (pp. 32-33). Il va sans dire que ce milieu n'était pas particulièrement perméable au syndicalisme (chap. 3, «Years of Survival»): "The Labor force consisted of ethnic and national minorities, immigrants who were either too beaten or too ambitious to support a union. They were trapped by a mode of production that camouflaged the employer and defied their efforts to locate the center of industrial responsibility» (p. 40).

Ce n'est qu'après plusieurs tentatives inefficaces que l'ILGWU est formée en 1900 ; elle connaît sa première grève importante - qui dure 2 mois - en 1909, (chap. 4, "The Shirtwaist Makers' Strike, $1909 »)$. Elle est suivie d'une autre grève déterminante qui se règle par la conclusion d'un accord collectif s'appliquant à l'ensemble de l'industrie (chap. 5, "The Cloakmakers' Strike, 1910 » et chap. 6 "The Protocol of Peace: its Rise»). Cet accord assurait l'existence de l'ILGWU et précisait le champ du négociable: échelle de salaires, modes d'arbitrage des conflits, conditions minimales de travail, etc. L'accord sera abrogé en 1916. (chap. 7, «The Protocol of Peace; Its Decline»). Il aura été l'occasion pour le syndicat de déborder les revendications strictement salariales et de se sensibiliser à des questions comme celles de la santé, de l'éducation et de l'action politique (chap. 8, "Life and Death» et chap. 11, «Way of Life»).

L'ILGWU subit les contrecoups de la crise économique pendant les années 1930; elle revit avec le «New Deal» du président Roosevelt qui entraine de plus grandes possibilités d'organisation syndicale (chap. 9, «Rebirth of the Union» et chap. 10 «The New Deal»). Depuis l'ILGWU est devenu un syndicat puis- 
sant qui a débordé les frontières américaines; au Québec, il regroupe près de 20,000 syndiqués à l'intérieur de l'Union internationale des ouvriers du vêtement pour dames.

M. Stein, qui est membre de l'ILGWU depuis 50 ans et qui édite son journal a fait un choix de textes qui constituent un hommage aux pionniers du syndicalisme dans le vêtement. Un historien aurait sûre- ment procédé autrement mais la variété et la qualité des textes retenus donnent une vision fort crédible des conditions de travail et des difficultés d'introduire le syndicalisme dans le monde des "sweatshop", ce qui en fait une bonne contribution à l'histoire du travail en Amérique.

Gilles DUSSAULT

Université Laval

\title{
THE JOURNAL OF INDUSTRIAL RELATIONS
}

\author{
The Journal of the Industrial Relations Society of Australia \\ Editor: John Niland, University of New South Wales
}

Vol. 20, No. 1, March 1978.

NEAT - A Little More Than an Acronym: Julian Teicher; Political Strikes: Three Burning Questions: P. R. Hay; Over-Awards: Bargaining at Plant Level in Australia: Paul Sutcliffe and Margaret Gardner; The Federal Law Relating to Union Amalgamation in Australia: Help or Hindrance: Dlaniel Khoury; National Wage Case Decisions in 1977: The Full Bench Survives But Yet Another Year: Braham Dabscheck; Decisions Affecting Industrial Relations in 1977: Jim Mahony; Australian Trade Unionism in 1977: Bob Carr; Employer Matters in 1977: R. N. Herbert; The ACTU Congress: A Comment: Milton Cockburn; An Assessment of Subsidised Relocation of Worker Policy in Britain: A Comment: Loren C. Scott, H. Tyrone B3lack, Lewis H. Smith and William A. Sirmon.

Four issues annually. Subscription, within Australia, is $\$ 17.00$ (Aust.) per annum, post free or $\$ 5.00$ (Aust.) post free for single copies. Overseas sea mail surcharge of $\$ 3.00$ (Aust.) per annum or an additional $\$ 14.00$ (Aust.) per annum for airmail. Order from: G. J. Bennett Associates, P.D. Box 2260 G.P.O., Sydney, Australia 2001. 